\title{
Edge Profile Effect of Tunnel Oxide on Erase Threshold-Voltage Distributions in Flash Memory Cells
}

\author{
Bomsoo Kim, Wook-Hyun Kwon, Member, IEEE, Chang-Ki Baek, Younghwan Son, \\ Chan-Kwang Park, Member, IEEE, Kinam Kim, Fellow, IEEE, and Dae M. Kim
}

\begin{abstract}
The erase threshold-voltage $\left(V_{T}\right)$ distribution in Flash electrically erasable programmable read-only memory cells was investigated versus the tunnel oxide edge profiles in selfaligned shallow trench isolation (SA-STI) and self-aligned poly (SAP) cells. The capacitive coupling with offset voltage correction is transcribed into $V_{T}$ transient for simulating erase $V_{T}$ dispersion without numerous full structure device simulations. It is shown that SAP gives rise to smaller $V_{T}$ dispersion, compared with SA-STI. The $V_{T}$ dispersion resulting from variations in dielectric thickness and oxide edge profiles is shown to fall far short of observed $V_{T}$ distribution, calling for examination of additional process and cell parameters.
\end{abstract}

Index Terms-Capacitive coupling equation with offset voltage, edge profile effect, erase threshold voltage distribution, Flash electrically erasable programmable read-only memory (EEPROM) cell.

\section{INTRODUCTION}

A CRITICAL concern in Flash electrically erasable programmable read-only memory cells is the tight control of $V_{T}$ within given specifications, especially in multilevel cells [1]. As the cell size gets smaller and the density becomes larger, the small perturbations in processing and operation of the cell give rise to large $V_{T}$ distributions. Even in fresh virgin cells, the $V_{T}$ distribution is generally wide, due to the effects of process variations being amplified upon the shrunken cell geometry. $V_{T}$ further shifts and broadens due to repeated program/erase cycles and trap assisted tunneling or charge loss during retention [2], [3].

$V_{T}$ dispersion in small cells is more sensitively affected by local effects such as the discrete dopant fluctuation [4]-[7], random polysilicon grain size [8], etc., Also, because of the high packing density the interference between adjacent cells results in further $V_{T}$ shifts [9]. Recently, the control of the edgeprofile of the tunnel oxide in 90-nm NOR Flash memory cells was shown to improve $V_{T}$ distribution [10], [11], indicating that the edge geometry also affects $V_{T}$.

Manuscript received June 6, 2006; revised August 18, 2006. This work was supported by Samsung Electronics, Inc. under a research grant. The review of this paper was arranged by Editor V. R. Rao.

B. Kim, C.-K. Baek, Y. Son, and D. M. Kim are with the School of Computational Sciences, Korea Institute for Advanced Study (KIAS), Seoul 130-722, Korea.

W.-H. Kwon, C.-K. Park, and K. Kim are with the Advanced Technology Development Team, Semiconductor R\&D Center, Samsung Electronics Company, Ltd., Gyeonggi-Do 449-711, Korea.

Color versions of Figs. 3, 4, and 9 are available at http://ieeexplore.ieee.org.

Digital Object Identifier 10.1109/TED.2006.885101
In downsizing the cell, its width constitutes the critical dimension. The large drain voltage required for the channel hot electron injection in NOR-type cells renders the cell vulnerable to the punchthrough effect, preventing the channel length reduction. In contrast, shrinking the channel width is free of such constraints and has the additional advantage of low programming current. In a narrow channel width, however, the edge effects of tunnel oxide become prominent such as the electric field crowding, affecting gate current and thus $V_{T}$.

In Section II, the erase $V_{T}$ distribution due to the onedimensional uniform variation of oxide-nitride-oxide (ONO) or tunnel oxide thickness is analytically discussed, giving an insight into the $V_{T}$ dispersion. The $V_{T}$ transient equation derived from the capacitive couplings with the offset voltage correction is shown to describe $V_{T}$ distributions, when the specific process variations are embedded into the general cell parameters. In Section III, a fast simulation of the erase $V_{T}$ distribution due to the oxide edge effects associated with self-aligned shallow trench isolation (SA-STI) [12] and self-aligned poly (SAP) [13] is performed without resorting to the full structure device simulations, and a few conclusions are drawn in Section IV.

\section{BACKGROUND DISCUSSION}

\section{A. Capacitive Coupling Equation and $V_{\text {offset }}$}

The well-known capacitive coupling equation [14] generally has additional offset voltage terms ( $\left.V_{\text {offset }}\right)$, which assumes different values depending on channel accumulation or inversion due to the work function difference between the terminals and channel depletion capacitance [15], [16]

$$
V_{\mathrm{FG}}=\alpha_{\mathrm{CG}} V_{\mathrm{CG}}+Q_{\mathrm{FG}} / C_{T}+V_{\text {offset }} .
$$

Here, the drain, source, and bulk terms are deleted since they are always grounded in this paper. The Gauss law applied to the floating-gate terminal yields $V_{\text {offsetI }}=\left(V_{\mathrm{FB}}+2 \phi_{B}\right) \times$ $C_{\mathrm{TNL}} / C_{T}$ and $V_{\mathrm{offset} A}=V_{\mathrm{FB}} \times C_{\mathrm{TNL}} / C_{T}$ in channel inversion and accumulation, respectively. Here, $V_{\mathrm{FB}}$ is the flatband voltage between the floating-gate and the bulk, $C_{\mathrm{ONO}}$ and $C_{\mathrm{TNL}}$ the interpoly and tunnel oxide capacitances (cf. $C_{T}=$ $C_{\mathrm{ONO}}+C_{\mathrm{TNL}}$ ), and $\phi_{B}$ is the bulk Fermi potential so that $2 \phi_{B}$ represents the surface bending at the onset of strong inversion. 


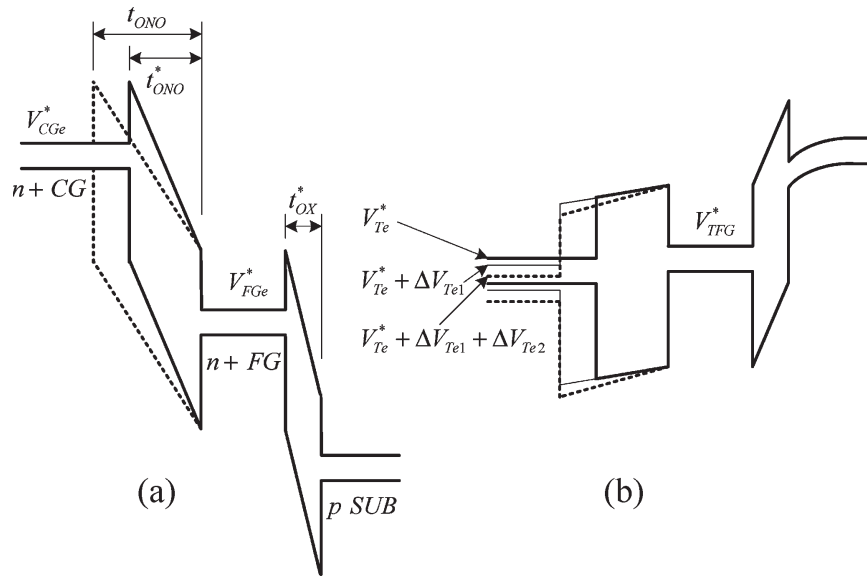

Fig. 1. (a) Band diagram in Flash memory cells with on- and off-target ONO thickness, in erase where a negative bias is applied at the control gate and (b) in reading where the floating-gate voltage is fixed at given $V_{\mathrm{TFG}}$.

\section{B. Erase $V_{T}$ versus Uniform Dielectric Thickness Variation}

As the first step for understanding the erase $V_{T}$ distribution, consider the effect of uniform variation of ONO thickness $\left(t_{\mathrm{ONO}}\right)$ from its target value $\left(t_{\mathrm{ONO}}^{*}\right)$ across the gate electrode. Fig. 1(a) shows the energy band diagram in erase, drawn in solid and dashed lines for on-target $\left(t_{\mathrm{ONO}}^{*}\right)$ and off-target $\left(t_{\mathrm{ONO}}\right)$ cells, respectively. Here, use has been made of the fact that the tunnel oxide electric field assumes the same value at the end of erase [3], regardless of the value of programmed $V_{T}$ or ONO thickness. This property is detailed in Appendix. Since the tunnel oxide electric fields are the same for the case of both $t_{\mathrm{ONO}}$ and $t_{\mathrm{ONO}}^{*}$, the difference in the stored charge in the floating-gate is given by

$$
\Delta Q_{\mathrm{FG}}=\left(C_{\mathrm{ONO}}-C_{\mathrm{ONO}}^{*}\right)\left(V_{\mathrm{FGe}}^{*}-V_{\mathrm{CGe}}^{*}\right)
$$

where $C_{\mathrm{ONO}}^{*}$ and $C_{\mathrm{ONO}}$ are the ONO capacitances in on- and off-target cells and $V_{\mathrm{FGe}}^{*}$ and $V_{\mathrm{CGe}}^{*}$ are the common floating and control gate voltages [see Fig. 1(a)].

The erase $V_{T}$ is affected by both $\Delta t_{\mathrm{ONO}}\left(=t_{\mathrm{ONO}}-t_{\mathrm{ONO}}^{*}\right)$ and $\Delta Q_{\mathrm{FG}}$. To estimate the change in $V_{T}$, first introduce $\Delta V_{\mathrm{Te} 1}$ due to $\Delta t_{\mathrm{ONO}}$, while putting $\Delta Q_{\mathrm{FG}}=0$. In this case, the electric field in ONO is the same in both cells, as shown in Fig. 1(b) and $\Delta V_{\mathrm{Te} 1}$ is thus given by

$$
\Delta V_{\mathrm{Te} 1}=\left(\Delta t_{\mathrm{ONO}} / t_{\mathrm{ONO}}^{*}\right)\left(V_{\mathrm{Te}}^{*}-V_{\mathrm{TFG}}^{*}\right)
$$

where $V_{\mathrm{Te}}^{*}$ is the threshold voltage at the control gate after erase, and $V_{\mathrm{TFG}}^{*}$ is that at floating-gate.

Next, $\Delta V_{\mathrm{Te} 2}$ due to $\Delta Q_{\mathrm{FG}}$ can be found by using (2) and $C_{\mathrm{ONO}} / C_{\mathrm{ONO}}^{*}=t_{\mathrm{ONO}}^{*} / t_{\mathrm{ONO}}$

$$
\begin{aligned}
\Delta V_{\mathrm{Te} 2} & \equiv-\Delta Q_{\mathrm{FG}} / C_{\mathrm{ONO}} \\
& =\left(t_{\mathrm{ONO}} / t_{\mathrm{ONO}}^{*}-1\right)\left(V_{\mathrm{FGe}}^{*}-V_{\mathrm{CGe}}^{*}\right) .
\end{aligned}
$$

The total $V_{T}$ variation $\Delta V_{\mathrm{Te}}=\Delta V_{\mathrm{Te} 1}+\Delta V_{\mathrm{Te} 2}$ is thus given by

$$
\Delta V_{\mathrm{Te}}=\left(\Delta t_{\mathrm{ONO}} / t_{\mathrm{ONO}}^{*}\right)\left(V_{\mathrm{Te}}^{*}-V_{\mathrm{TFG}}^{*}+V_{\mathrm{FGe}}^{*}-V_{\mathrm{CGe}}^{*}\right) .
$$

Here, $V_{\mathrm{TFG}}^{*}$ and $V_{\mathrm{FGe}}^{*}$ are implicit voltages and can be replaced with the known voltages $V_{\mathrm{Te}}^{*}, V_{\mathrm{CGe}}^{*}$ using (1) for both channel inversion and accumulation, obtaining

$$
\Delta V_{\mathrm{Te}} \approx \frac{\Delta t_{\mathrm{ONO}}}{t_{\mathrm{ONO}}^{*}}\left(1-\alpha_{\mathrm{CG}}^{*}\right)\left(V_{\mathrm{Te}}^{*}-V_{\mathrm{CGe}}^{*}\right)
$$

One can also analyze the erase $V_{T}$ change due to the uniform variation of tunnel oxide thickness $\Delta t_{\mathrm{OX}}$ in a similar manner, obtaining an expression symmetric with respect to (6)

$$
\Delta V_{\mathrm{Te}} \approx \frac{\Delta t_{\mathrm{OX}}}{t_{\mathrm{OX}}^{*}} \alpha_{\mathrm{CG}}^{*}\left(V_{\mathrm{Te}}^{*}-V_{\mathrm{CGe}}^{*}\right) .
$$

In (6) and (7), $\Delta V_{\mathrm{Te}}$ is characterized in terms of $\Delta t_{\mathrm{ONO}} / t_{\mathrm{ONO}}^{*}, \Delta t_{\mathrm{OX}} / t_{\mathrm{OX}}^{*}$ and operating voltages. Clearly, the $V_{T}$ variation can be made small by reducing $V_{\mathrm{Te}}^{*}$ and $V_{\mathrm{CGe}}^{*}$ but reducing $V_{\mathrm{Te}}^{*}$ is limited by the overerase margin, while small $V_{\mathrm{CGe}}^{*}$ necessitates long erase time. Also, since the variations, $\Delta t_{\mathrm{ONO}} / t_{\mathrm{ONO}}^{*}$ and $\Delta t_{\mathrm{OX}} / t_{\mathrm{OX}}^{*}$ are basically unknown quantities, it appears reasonable to keep $\alpha_{\mathrm{CG}}^{*}$ at about 0.5 . In this case, for typical values of $V_{\mathrm{Te}}^{*}=3 \mathrm{~V}, V_{\mathrm{CGe}}^{*}=-17 \mathrm{~V}, \Delta t_{\mathrm{ONO}} / t_{\mathrm{ONO}}^{*}$ or $\Delta t_{\mathrm{OX}} / t_{\mathrm{OX}}^{*}$ of $\pm 5 \%$ is shown to give the erase $V_{T}$ shift of $\Delta V_{\mathrm{Te}} \sim \pm 0.5 \mathrm{~V}$ centered at $3 \mathrm{~V}$.

Next, the erase $V_{T}$ change due to the nonuniform local effect such as the edge profile of the tunnel oxide is examined in the following section.

\section{Transient $V_{\mathrm{FG}}$ and $V_{T}$ During Erase}

The time-dependent equations of $V_{\mathrm{FG}}$ and $V_{T}$ during the erase are derived from the basic capacitive coupling. These equations can quantify the transient $V_{\mathrm{FG}}$ and $V_{T}$ versus the process variations. The analytical solution of $V_{\mathrm{FG}}$ and $V_{T}$ transients were derived in terms of specific cell parameters in [15]. However, the transient equations presented here are specified by more general set of parameters, so that various process variations, e.g., the local edge effects of tunnel oxide, can be easily embedded into each parameter.

In the present approach $V_{\mathrm{FG}}, V_{T}$ transients are analyzed by switching back and forth between erase and read modes. During the erase, in which the control gate is constantly biased at $V_{\mathrm{CGe}}$ while other terminals are grounded, the excess charge $Q_{\mathrm{FG}}$ in the floating-gate is tunneled out as gate current, $I_{G}$, i.e., $d Q_{\mathrm{FG}} / d t=I_{G}$. Here, $I_{G}$ depends on changing $V_{\mathrm{FG}}$, viz., $I_{G}=I_{G}\left(V_{\mathrm{FG}}\right)$. Since the cell terminals are all kept constant during the erase, differentiating (1) in accumulation with time yields [17]

$$
\frac{d V_{\mathrm{FG}}}{d t}=\frac{1}{C_{T}} \cdot \frac{d Q_{\mathrm{FG}}}{d t}=\frac{I_{G}\left(V_{\mathrm{FG}}\right)}{C_{T}} .
$$

The initial value of $V_{\mathrm{FG}}(t)$ is obtained from (1) in both accumulation and inversion, operative, respectively, at the beginning of erase and read at the end of programming. In this case, $Q_{\mathrm{FG}}$ is the same and by subtracting (1) in inversion from (1) in accumulation there results with $\alpha_{\mathrm{CG}}=C_{\mathrm{ONO}} / C_{T}$

$$
V_{\mathrm{FG} 0}=V_{\mathrm{TFG}}+\frac{C_{\mathrm{ONO}}}{C_{T}}\left(V_{\mathrm{CGe}}-V_{T 0}\right)+\Delta V_{\mathrm{offset}} .
$$




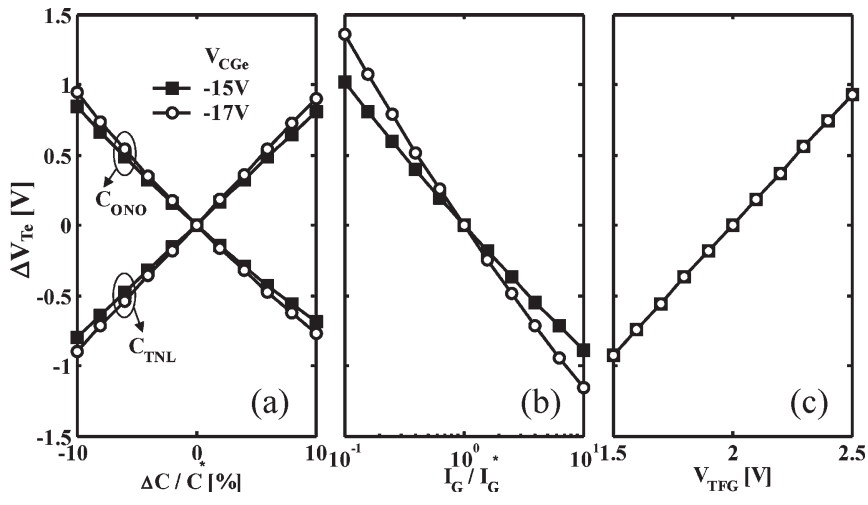

Fig. 2. (a) Erase $V_{T}$ versus nominal process parameter variation with respect to the target values: interpoly and tunnel oxide capacitances, $C_{\mathrm{ONO}}, C_{\mathrm{TNL}}$; (b) tunneling gate current, $I_{G}$; (c) threshold voltage of floating-gate, $V_{\mathrm{TFG}}$.

Here, $V_{\mathrm{FG} 0}$ is the initial $V_{\mathrm{FG}}$ in erase and $V_{T 0}$ the user-definable initial $V_{T}$ at the control gate in erase, or equivalently final $V_{T}$ at the end of programming and $\Delta V_{\text {offset }}=V_{\text {offset } A}-V_{\text {offsetI }}$.

In reading mode, $V_{\mathrm{TFG}}$ is, by definition, fixed at a value determined solely by the onset of strong inversion, while $V_{T}$ at the control gate changes. Thus, by differentiating (1) in inversion with respect to time one finds

$$
0=\alpha_{\mathrm{CG}} \frac{d V_{T}}{d t}+\frac{1}{C_{T}} \cdot \frac{d Q_{\mathrm{FG}}}{d t}
$$

(10) and (8) provide a linear relationship between $\Delta V_{T}$ and $\Delta V_{\mathrm{FG}}$

$$
\Delta V_{T}=-\frac{1}{\alpha_{\mathrm{CG}}} \Delta V_{\mathrm{FG}} .
$$

Thus, the change in time of $V_{T}$ from $V_{T 0}$ is given in terms of that of $V_{\mathrm{FG}}$ from $V_{\mathrm{FG} 0}$. Now, $V_{\mathrm{FG} 0}$ and $V_{T 0}$ are related by (9) and one can write from (9) and (11)

$$
V_{T}(t)=V_{\mathrm{CGe}}-\frac{C_{T}}{C_{\mathrm{ONO}}}\left[V_{\mathrm{FG}}(t)-V_{\mathrm{TFG}}-\Delta V_{\mathrm{offset}}\right] .
$$

The set of equations (8), (9), and (12) makes it possible to simulate the transient behavior of $V_{\mathrm{FG}}$ and $V_{T}$ in terms of the pertinent cell parameters $\left(C_{\mathrm{ONO}}, C_{\mathrm{TNL}}, I_{G}\right.$, and $\left.V_{\mathrm{TFG}}\right)$. Any random process variation is to be represented in corresponding variation of one of these parameters. For example, the discrete dopant fluctuation causes $V_{\text {TFG }}$ variation, while the polysilicon grain size affects $I_{G}$. Also, the nonuniform edge profile alters both $I_{G}$ and $C_{\mathrm{TNL}}$.

The contribution of these parameters to the erase $V_{T}$ distribution, $\Delta V_{\mathrm{Te}}$ can be calculated by varying each parameter, while keeping others fixed to the target values and by solving (8), (9), and (12). Fig. 2 shows the result obtained versus the cell parameter variations for two different erase voltages, $V_{\mathrm{CGe}}=$ $-15 \mathrm{~V}$ and $-17 \mathrm{~V}$. In the calculation, $V_{\mathrm{Te}}^{*}$ is set to $3 \mathrm{~V}$, and the erase time was set to $90 \mathrm{~ms}$ or $1.3 \mathrm{~ms}$ for $V_{\mathrm{CGe}}=-15 \mathrm{~V}$ or $-17 \mathrm{~V}$, respectively. Also, $I_{G}^{*}$ was measured from an array pattern and $V_{\mathrm{TFG}}^{*}$ was found from unit reference cell where floating and control gates are shorted. Also, $C_{\mathrm{ONO}}^{*}$ and $C_{\mathrm{TNL}}^{*}$ were obtained by simulating TEM cross section [10]. These target parameters are slightly altered to fit the observed erase $V_{T}$ versus time, resulting in $V_{\mathrm{TFG}}^{*}=2 \mathrm{~V}, C_{\mathrm{ONO}}^{*}=5.13 \times$ $10^{-16} \mathrm{~F} / \mu \mathrm{m}$, and $C_{\mathrm{TNL}}^{*}=4.37 \times 10^{-16} \mathrm{~F} / \mu \mathrm{m}$ (normalized in length).

As clear from Fig. 2(a), the relative variations, $\Delta C_{\mathrm{ONO}} / C_{\mathrm{ONO}}^{*}, \Delta C_{\mathrm{TNL}} / C_{\mathrm{TNL}}^{*}$ by $10 \%$ is shown to give about $\Delta V_{T} \approx 1 \mathrm{~V}$ with larger $V_{\mathrm{CGe}}$ inducing slightly greater change. These results are consistent with the predictions of (6) or (7). Also, $I_{G}$ variation by an order of magnitude centered at $I_{G}^{*}$ results in $\Delta V_{T} \approx 1 \mathrm{~V}$ [Fig. 2(b)]. Finally, $\Delta V_{\mathrm{TFG}}$ by say $0.5 \mathrm{~V}$ gives rise to $\Delta V_{\mathrm{Te}}$ by about $1 \mathrm{~V}$, corresponding to about $\Delta V_{\mathrm{TFG}} / \alpha_{\mathrm{CG}}$ [Fig. 2(c)]. It is interesting to note that Fig. 2 provides a simple means by which to single out the dominant cause of $\Delta V_{\mathrm{Te}}$ by measuring the $V_{T}$ distribution using different erase voltage at the control gate $V_{\mathrm{CGe}}$. For example, if $V_{T}$ distribution has an appreciable dependence on $V_{\mathrm{CGe}}$, the dominant cause is $I_{G}$ variation, while a weak dependence points to the dominance of $V_{\mathrm{TFG}}$ variation.

The erase $V_{T}$ variations in Fig. 2 are approximately linear with respect to the input parameters and the total $V_{T}$ distribution can therefore be estimated by $\sigma_{\text {total }}^{2}=\sigma_{\text {CONO }}^{2}+\sigma_{\text {CTNL }}^{2}+$ $\sigma_{I G}^{2}+\sigma_{\mathrm{VTFG}}^{2}$, where $\sigma$ is the width of $V_{T}$ distribution. The total distribution can also be calculated directly from (8), (9), and (12) by randomly varying the parameters simultaneously. Now, that $\Delta V_{\mathrm{Te}}$ has been specified via the random variations of cell parameters, the next task is to discuss the origins of such variation. In Section III, the edge profile of the gate oxide is discussed as an example.

\section{ERASE $V_{T}$ Distribution Versus Oxide EdGE PROFILE}

To simulate the erase $V_{T}$ distribution versus the tunnel oxide edge profile, one could perform the transient device simulation incorporating a large number of randomly generated profiles in conjunction with control (CG) and floating (FG) gates, ONO and tunnel oxide (OX), and the substrate (SUB). In this paper, this direct simulation is bypassed in favor of two time-saving measures. First, the device simulations are confined to the FG-OX-SUB structure and the gate current $\left(I_{G}\right)$ and tunnel oxide capacitance $\left(C_{\mathrm{TNL}}\right)$ associated therein. Then, the transient simulation of the cell is performed for each different edge profile by using (8), (9), and (12), with $I_{G}$ and $C_{\mathrm{TNL}}$ explicitly inserted. By bypassing the full structure simulation, more mesh nodes can be assigned to the edge region for accurate simulation of electric field and $I_{G}$, giving thereby the benefits to the threedimensional simulation. Second, a meaningful simulation of $V_{T}$ distribution requires sample numbers stretching to several orders, and prohibitively large simulation time. This difficulty is circumvented by simulating in advance specific numbers of sample and incorporating other samples via interpolation within the sample matrix, to be detailed later.

Fig. 3 depicts the cross-sectional edge structure along the width in SA-STI [Fig. 3(a)] and SAP [Fig. 3(b)] processed cells. The parameters of the target cells are $t_{\mathrm{OX}}$ at the center of $85 \AA$, and cell width $(W)$ of $90 \mathrm{~nm}$ and the substrate doping of $N_{A}=2 \times 10^{18} \mathrm{~cm}^{-3}$. $I_{G}$ was simulated versus $V_{\mathrm{FG}}$ and the tunnel oxide capacitance was extracted for use in (8), (9), 

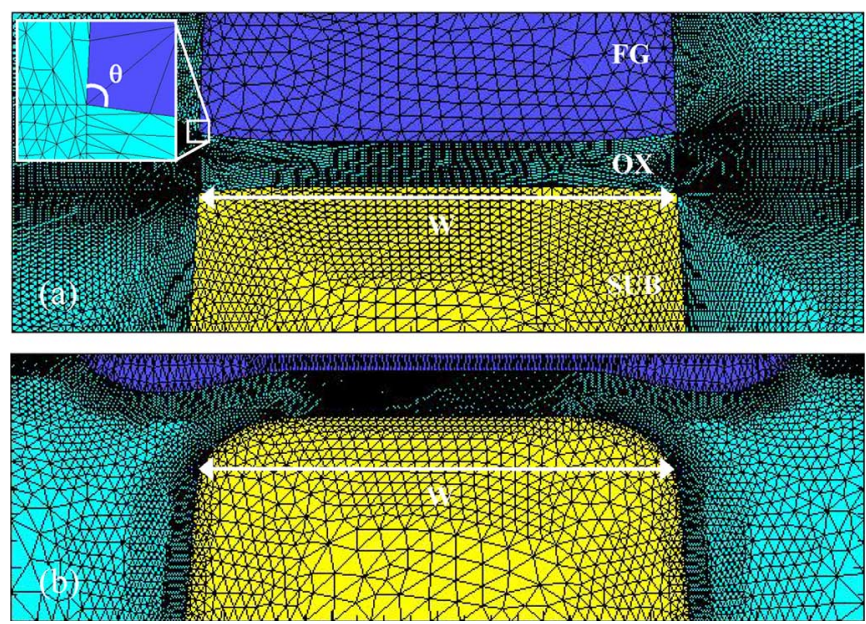

Fig. 3. Cross-sectional views of the floating-gate-tunnel oxide-substrate structures in (a) SA-STI and (b) SAP cells.

and (12). A particular attention was paid to the mesh formation near the edge in SA-STI, since the tunneling current depends sensitively on the mesh size around the edge boundaries. This apparent errorlike mesh dependence is due to the angle $(\theta)$ at the edge remaining unchanged regardless of the mesh size [see the inset of Fig. 3(a)]. This implies a different radius of curvature at the edge for different mesh size. For the case of SAP, there was no such mesh-dependent anomaly, and the material boundaries are sufficiently smooth to render both the mesh lines on the boundary and the angle between them conform to the boundaries.

In this paper, the boundary conforming to the small radius of curvature was not used, since it is difficult to deal with in Medici [18] mesh generator. Rather, an ideal edge profile shown in the inset of Fig. 3(a) was used, with the same topology of mesh triangulations for all samples to avoid the mesh-dependent $I_{G}$ fluctuation. The mesh size was chosen to be $3 \AA$ in the oxide near the edge for accommodating the abrupt change of electric field, and to be $2 \mathrm{~nm}$ on the edge boundaries for relieving the extremely high electric field and high tunneling current by way of averaging the electric field in the mesh element.

Fig. 4 shows the simulation flow of the erase $V_{T}$ distribution. Here, the boundary files for specified variation were generated by selecting tunnel oxide thickness $\left(\Delta T_{\mathrm{OX}}\right)$, edge thickness $(\Delta E)$, and cell width $(\Delta W)$ as random process variations [Fig. 4(a)]. Each variation was ranged in common from -10 to $10 \AA$, and seven samples were selected at regular intervals, so that the matrix of $7 \times 7 \times 7$ samples was selected for simulation [Fig. 4(b)]. Both $I_{G}$ and $C_{\text {TNL }}$ were simulated in these samples with erase $V_{T}$ s calculated using (8), (9), and (12) [Fig. 4(c)]. $V_{\mathrm{TFG}}$ change was assumed to be caused only by $\Delta T_{\mathrm{OX}}$. For erase $V_{T}$ distribution, $512 \mathrm{~K}$ samples were randomly selected following the Gaussian distribution from the sample matrix [Fig. 4(d)] and the erase $V_{T}$ s were computed by interpolating $V_{T}$ versus $\Delta T_{\mathrm{OX}}, \Delta E$, and $\Delta W$ in Fig. 4(c). Naturally, $V_{T}$ variation should be smooth in the sample matrix, so that the interpolation gives reliable result using an adequate number of samples chosen within the variation range. This requirement was met in the sample matrix chosen.
Fig. 5 shows $I_{G}, C_{\mathrm{TNL}}$, and $V_{T}$ versus $\Delta T_{\mathrm{OX}}, \Delta E$, or $\Delta W$ for both SA-STI and SAP. These results were obtained by varying only one parameter while keeping others fixed. Clearly, $I_{G}$ is shown to vary significantly in SA-STI cells with $\Delta T_{\mathrm{OX}}$, $\Delta E$, and $\Delta W$, while SAP processed cells exhibit a large $I_{G}$ variation only with $\Delta T_{\mathrm{OX}}$ [Fig. 5(a)]. The behavior of $C_{\mathrm{TNL}}$ is roughly same in both cells, undergoing a large variation with $\Delta T_{\mathrm{OX}}$ [Fig. 5(b)]. The change of $V_{\mathrm{TFG}}$ due to $\Delta T_{\mathrm{OX}}$ is same for both process types. With $I_{G}, C_{\mathrm{TNL}}$, and $V_{\mathrm{TFG}}$ thus obtained, the erase $V_{T}$ was calculated by using (8), (9), and (12). The common parameters used for calculation are: $V_{\mathrm{TFG}}^{*}=2 \mathrm{~V}, V_{\mathrm{CGe}}=-17 \mathrm{~V}, \phi_{B}=0.48 \mathrm{~V}$, and $V_{T 0}=9 \mathrm{~V}$. Also, $C_{\mathrm{ONO}}$ was chosen such that $\alpha_{\mathrm{CG}}=0.54$ at the target sample and the erase $V_{T}$ at the target sample was chosen to be $3 \mathrm{~V}$ by adjusting the erase time in both cells. As clear from Fig. 5(c) the dominant parameters affecting $V_{T}$ are the variation of the edge profile $(\Delta E)$ and the tunneling oxide thickness $\left(\Delta T_{\mathrm{OX}}\right)$ in SA-STI and SAP samples, respectively.

Fig. 6 shows the simulated $V_{T}$ distributions of SA-STI and SAP cells, obtained by sampling $512 \mathrm{~K}$ cells. The $3 \sigma$ random variations of $\Delta T_{\mathrm{OX}}, \Delta E$, and $\Delta W$ were fixed at 1,1 , and $3 \AA$, respectively. As clear from Fig. 6, $V_{T}$ in SA-STI cells is shown sensitively affected by edge profile parameters, and a large $V_{T}$ distribution ensues due to the $3 \sigma$ random variations of $\Delta E$ and $\Delta W$. On the other hand, the $V_{T}$ distribution of SAP cells is mainly determined by $\Delta T_{\mathrm{OX}}$, and is nearly independent of the edge profile variations. Since the controllability of the edge region is in general difficult, SA-STI processed cells are expected to have a broader $V_{T}$ distribution, compared with SAP cells (see black and white square symbols in Fig. 6).

Fig. 7 shows the $V_{T}$ distributions measured from SA-STI and SAP samples. Clearly SAP cells have a width narrower by about $0.2-0.3 \mathrm{~V}$, compared to the former. However, the measured distributions are still much broader than those resulting from the edge profile effects discussed in Fig. 6. A quantitative comparison between the simulation and measured data was made as follows. First, a common "background distribution" was introduced for both cells, accounting for all possible process variations excluding the ones $\left(\Delta T_{\mathrm{OX}}, \Delta E\right.$, and $\left.\Delta W\right)$ in Fig. 4(a). Since these two cells are processed identically other than the edge profiling in the length direction it is reasonable to take the background distribution to be the same in both cells and analyze the edge effect separately as in Fig. 6. Combined to this background was the additional distribution arising from the edge effects simulated in Fig. 6 by using $3 \sigma_{\text {TOX }}=1 \AA$, $3 \sigma_{E}=3 \AA$, and $3 \sigma_{W}=3 \AA$.

It is important to point out that a fit to the data necessitates the introduction of a large background distribution, which in turn suggests additional parameters inherently operative and independent of the edge profile control contributing to the $V_{T}$ dispersion. This broad background distribution can be understood in part in terms of the random process variations of ONO, $\Delta V_{\mathrm{TFG}}$ by discrete dopant fluctuation being amplified by $1 / \alpha_{\mathrm{CG}}$ at the control gate with $\alpha_{\mathrm{CG}}$ itself varying from cell to cell, the cell-to-cell interference, random polygrain size, surface roughness, the edge effects along the cell width direction, etc.

Fig. 8 shows the standard deviation, $\sigma V_{T}$ of $V_{T}$ distributions as a function of the cell width, $W$. For $3 \sigma_{\text {TOX }}=1 \AA$, 

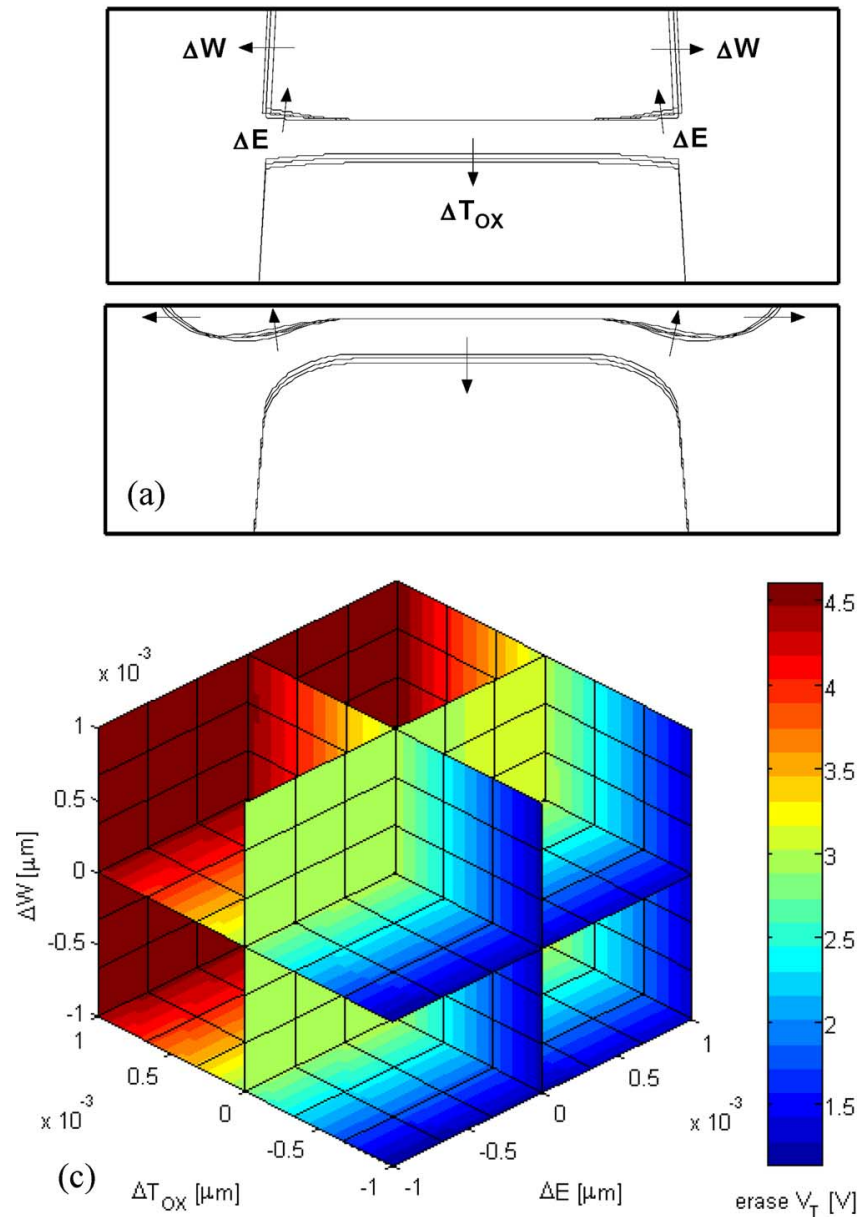

Fig. 4. Simulation flow of erase $V_{T}$ distribution.
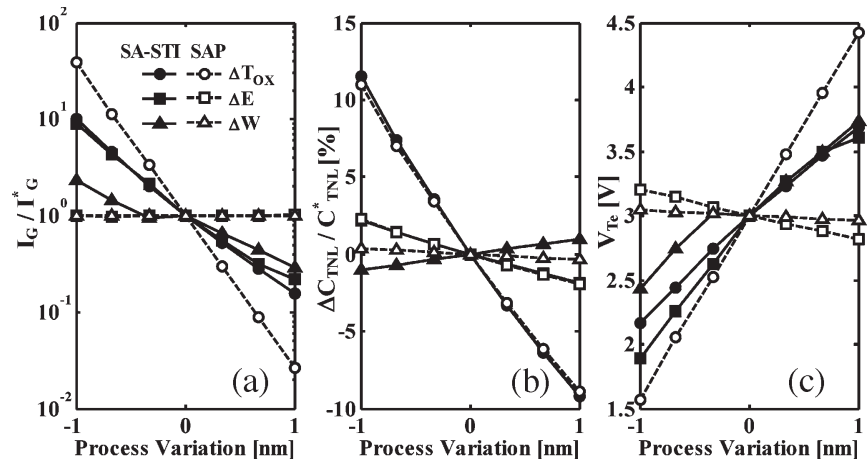

Fig. 5. Relative variations of (a) gate current and (b) tunnel oxide capacitance with respect to the target value, and the erase $V_{T}$ variation corresponding to (a) and (b) versus $\Delta T_{\mathrm{OX}}, \Delta E$, and $\Delta W$ in both SA-STI and SAP cells.

$3 \sigma_{E}=3 \AA$, and $3 \sigma_{W}=3 \AA, \sigma V_{T}$ of SA-STI cells tends to increase slightly with decreasing $W$. This is because the $V_{T}$ variation in this case is dominated by the edge profile effect while the reduction of the main tunneling region with decreasing $W$ plays an insignificant role. However, $\sigma V_{T}$ of SAP cells increases faster, as $W$ decreases and the main tunneling region is reduced therein. A highlight of Fig. 8 consists of the fact that within $W$ range considered, $\sigma V_{T}$ of SAP cells is smaller by a
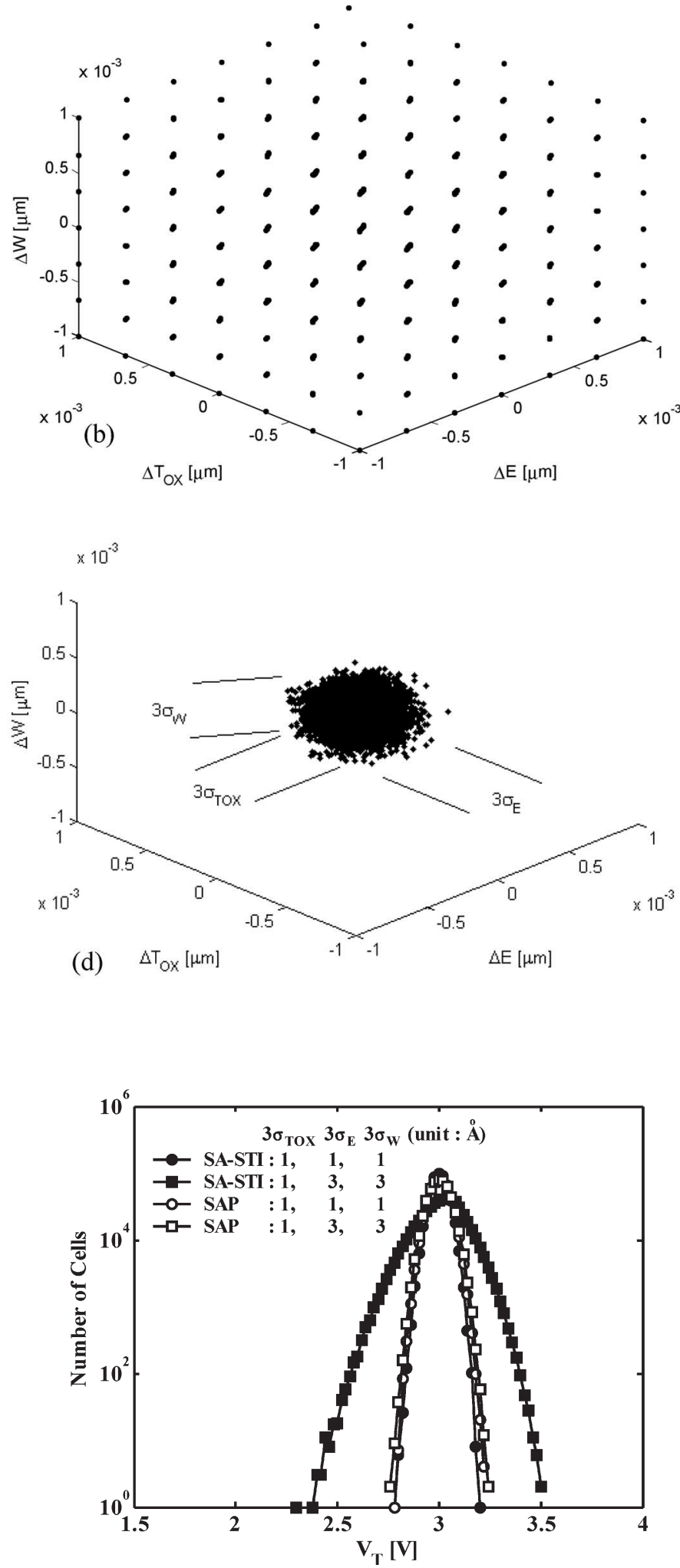

Fig. 6. Simulated erase $V_{T}$ distributions of SA-STI and SAP cells for different combinations of $\Delta T_{\mathrm{OX}}, \Delta E$, and $\Delta W$, showing the edge profile sensitivity of SA-STI cells.

factor 2, compared with SA-STI cells. This indicates that the SAP is suitable for cell scaling even if the edge profiling is to be controlled within 2- $\AA$ accuracy. As an additional note, $\sigma V_{T}$ of SAP cells is consistent with the prediction given by (7) as the edge effects are reduced and the cell width becomes longer. 


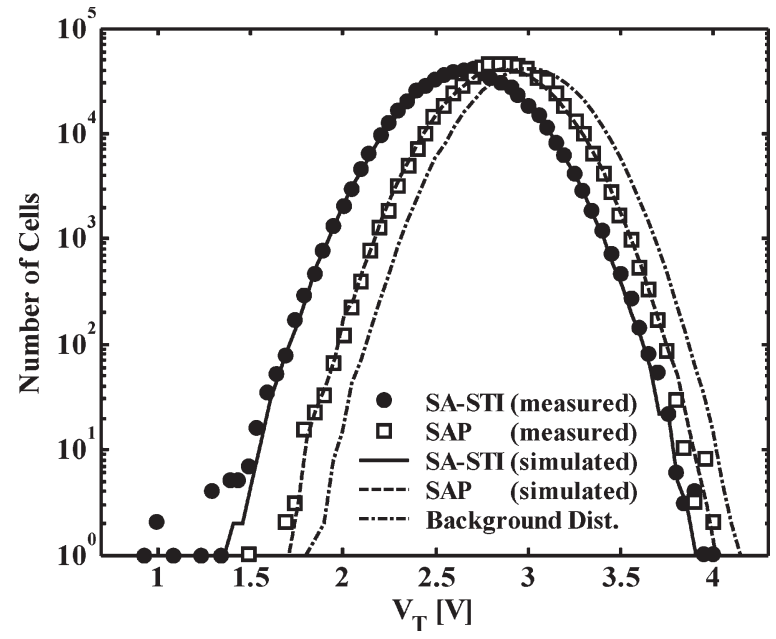

Fig. 7. Comparison of measured and simulated $V_{T}$ distributions of SA-STI and SAP cells.

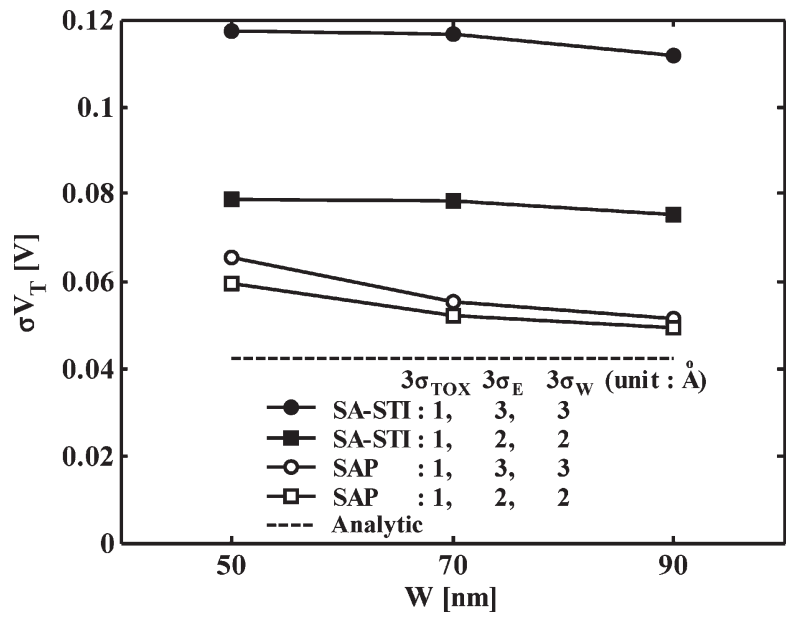

Fig. 8. Standard deviation of erase $V_{T}$ distributions of SA-STI and SAP cells versus the width.

\section{CONCLUSION}

The erase $V_{T}$ distribution in Flash memory cells was investigated as a function of the random process variations. Instead of the full-structure device simulations, the capacitive coupling equations with offset voltage correction were used to simulate the time dependent $V_{T}$ for different values of cell parameters $\left(C_{\mathrm{ONO}}, C_{\mathrm{TNL}}, I_{G}\right.$, and $\left.V_{\mathrm{TFG}}\right)$. Specific random process variation can be embedded into the cell parameters for analyzing its effect on erase $V_{T}$ distribution.

The method was applied to comparing two current process technologies, viz., SA-STI and SAP for the tunnel oxide edge effects on the erase $V_{T}$ distributions. The angled edge geometry in SA-STI cells with high tunneling current by electric field crowding near the edge region, combined with the relatively difficult control of edge profile, leads to wider $V_{T}$ dispersion than SAP cells. On the other hand, the SAP cells, relatively free of the edge effect are also shown to be more suitable for cell scaling. A good fit to the $V_{T}$ distribution measured from SASTI and SAP cells was shown to require a broad background $V_{T}$ distribution. This means that the tunnel oxide edge effects
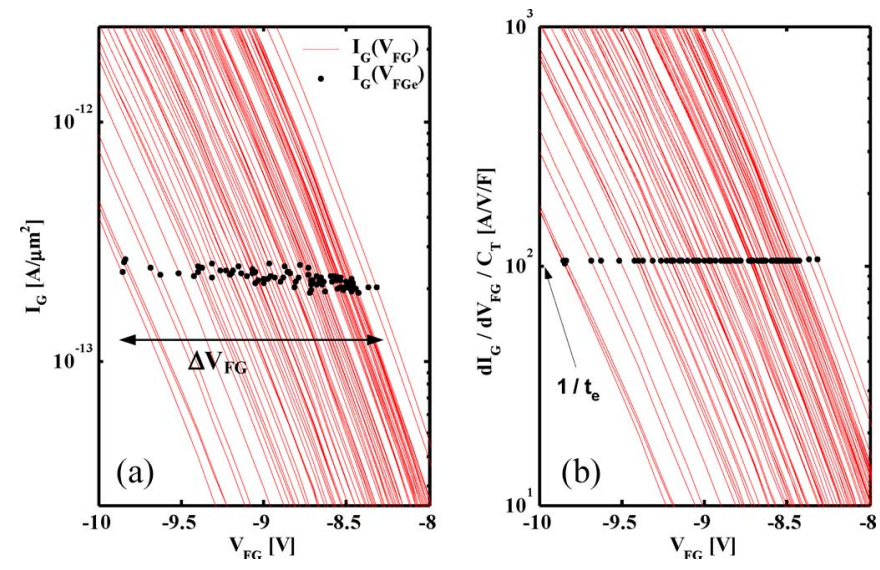

Fig. 9. (a) Family of gate currents generated to mimic $I_{G}$ from an ensemble of cells and the corresponding final floating-gate voltage found numerically at the end of erase (dots). (b) Corresponding plot calculated from (A.3).

constitute a mere fraction of the total observed distribution. It is therefore important to identify and further analyze these additional effects for optimizing the process parameters of small cell geometries.

\section{APPENDIX}

The FN tunneling gate current can be approximated via the first order Taylor expansion of $V_{\mathrm{FG}}$ near the final erase $V_{\mathrm{FG}}$, i.e., $V_{\mathrm{FGe}}$ as

$$
I_{G}=I_{G 0} \cdot e^{K \cdot V_{\mathrm{FG}}}
$$

where $I_{G 0}$ and $K$ are constants depending on $V_{\mathrm{FGe}}$ [3].

Under FN erase $V_{\mathrm{FG}}$ quickly decreases from its initial value $V_{\mathrm{FG} 0}$ and approaches the final $V_{\mathrm{FGe}}$ asymptotically, taking up most of the erase time $t_{e}$. Insert (A.1) into (8) and solve the differential equation, obtaining

$$
e^{-K \cdot V_{\mathrm{FGe}}}-e^{-K \cdot V_{\mathrm{FG} 0}}=\frac{K \cdot I_{G 0}}{C_{T}} t_{e} .
$$

Since $\left|V_{\mathrm{FGO}}\right|$ is much larger than $\left|V_{\mathrm{FGe}}\right|$ the second term of (A.2) is neglectable and one can write (A.2) as $K I_{\mathrm{Ge}} t_{e} / C_{T}=$ 1 with $I_{\mathrm{Ge}}=I_{G 0} \exp K V_{\mathrm{FGe}}$, regardless of any initial $V_{\mathrm{FG} 0}$. Since $K I_{\mathrm{Ge}}$ is the slope of (A.1) at $V_{\mathrm{FGe}}$, one can generalize (A.1) as

$$
I_{G}^{\prime}\left(V_{\mathrm{FGe}}\right) \cdot \frac{1}{C_{T}} \cdot t_{e}=1
$$

where $I_{G}^{\prime}=d I_{G} / d V_{\mathrm{FG}}$. (A.3) constitutes the condition determining the erase $V_{\mathrm{FG}}$ implicitly for given $t_{e}$.

Fig. 9(a) shows a family of FN tunneling currents. In each $I_{G}$ curve, (8) was solved numerically with given erase time of $t_{e}=10 \mathrm{~ms}$, obtaining $V_{\mathrm{FGe}}$. In generating a family of $I_{G}$ curves, $C_{T}$ was randomly varied around its target value to simulate the $I_{G} \mathrm{~S}$ at different memory cells. The resulting $V_{\mathrm{FGe}}$ is presented as a dot on each $I_{G}$ curve. Shown in Fig. 9(b) is corresponding $\left(d I_{G} / d V_{\mathrm{FG}}\right) / C_{T}$ together with $V_{\mathrm{FGe}}$ versus $V_{\mathrm{FG}}$. As clearly exhibited in Fig. 9(b), the trace of $V_{\mathrm{FGe}} \mathrm{s}$ accurately determines $1 / t_{e}$ for all curves considered, 
conclusively proving the validity of the termination condition (A.3) and the related assumptions. Also, in Fig. 9(a), the trace of $V_{\mathrm{FGe}} \mathrm{S}$ well approximates a straight line indicating constant $I_{G}$ as an approximated condition. Constant $I_{G}$ implies a constant electric field in the tunnel oxide, whose condition is already used in Section II-B and [3]. Finally, it is noted that $\Delta V_{\mathrm{Te}}$ due to varying $I_{G}$ can also be estimated via $\Delta V_{\mathrm{FG}} / \alpha_{\mathrm{CG}}$ with $\Delta V_{\mathrm{FG}}$ found in a manner as in Fig. 9(a).

\section{REFERENCES}

[1] B. Eitan, R. Kazerounian, A. Roy, G. Crisenza, P. Cappelletti, and A. Modelli, "Multilevel Flash cells and their trade-offs," in IEDM Tech. Dig., 1996, pp. 169-172.

[2] J.-D. Lee, J.-H. Choi, D. Park, and K. Kim, "Effects of interface trap generation and annihilation on the data retention characteristics of Flash memory cells," IEEE Trans. Device Mater. Rel., vol. 4, no. 1, pp. 110-117, Mar. 2004.

[3] N. Mielke, H. Belgal, I. Kalastirsky, P. Kalavade, A. Kurtz, Q. Meng, N. Righos, and J. Wu, "Flash EEPROM threshold instabilities due to charge trapping during program/erase cycling," IEEE Trans. Device Mater. Rel., vol. 4, no. 3, pp. 335-344, Sep. 2004

[4] A. Asenov, "Random dopant induced threshold voltage lowering and fluctuations in sub-0.1 $\mu \mathrm{m}$ MOSFET's: A 3-D 'atomistic' simulation study,' IEEE Trans. Electron Devices, vol. 45, no. 12, pp. 2505-2513, Dec. 1998

[5] A. Asenov, A. R. Brown, J. H. Davies, S. Kaya, and G. Slavcheva, "Simulation of intrinsic parameter fluctuations in decananometer and nanometer-scale MOSFETs," IEEE Trans. Electron Devices, vol. 50, no. 9, pp. 1837-1852, Sep. 2003.

[6] N. Sano, K. Matsuzawa, M. Mukai, and N. Nakayama, "Role of longrange and short-range Coulomb potentials in threshold characteristics under discrete dopants in sub-0.1 $\mu \mathrm{m}$ Si-MOSFETs," in IEDM Tech. Dig., 2000, pp. 275-278.

[7] T. Ezaki, T. Ikezawa, and M. Hane, "Investigation of realistic dopant fluctuation induced device characteristics variation for sub-100 nm CMOS by using atomistic 3D process/device simulator," in IEDM Tech. Dig., 2002, pp. 311-314.

[8] S. Muramatsu, T. Kubota, N. Nishio, H. Shirai, M. Matsuo, N. Kodama, M. Horikawa, S. Saito, K. Arai, and T. Okazawa, "The solution of overerase problem controlling poly-Si grain size-modified scaling principles for Flash memory," in IEDM Tech. Dig., 1994, pp. 847-850.

[9] W. H. Lee, "The effect of dimensional scaling on the erase characteristics of NOR Flash memory," IEEE Electron Device Lett., vol. 24, no. 4, pp. 245-247, Apr. 2003.

[10] W. H. Kwon, J. I. Han, B. Kim, C.-K. Baek, S.-P. Sim, W. H. Lee, J. H. Han, C. Jung, H. K. Lee, Y. K. Jang, J. H. Park, D. M. Kim, C.-K. Park, and K. Kim, "Highly reliable $256 \mathrm{Mb}$ NOR Flash MLC with self-aligned process and controlled edge profile," in Proc. Int. Conf. SSDM, Kobe, Japan, 2005, pp. 448-449.

[11] S.-P. Sim, W. H. Kwon, C. H. Lee, J. I. Han, W. H. Lee, C. Jung, H. K. Lee, Y. K. Jang, S. W. Park, J. H. Park, C.-K. Park, K. T. Kim, and K. Kim, "A 90 nm generation NOR Flash multilevel cell (MLC) with $0.44 / \mathrm{spl} \mathrm{mu} / \mathrm{m} / \mathrm{sup} 2 / /$ bit cell size," in Proc. IEEE VLSI-TSA Int. Symp. VLSI Tech., 2005, pp. 35-36.

[12] Y. Song, S. Lee, T. Kim, J. Han, H. Lee, S. Kim, J. Park, S. Park, J. Choi, J. Kim, D. Lee, M. Cho, K. Park, and K. Kim, "Highly manufacturable $90 \mathrm{~nm}$ NOR Flash technology with $0.081 / \mathrm{spl} \mathrm{mu} / \mathrm{m} / \mathrm{sup} 2 /$ cell size," in VLSI Symp. Tech. Dig., 2003, pp. 91-92.

[13] S. N. Keeney, "A $130 \mathrm{~nm}$ generation high density Etox Flash memory technology," in IEDM Tech. Dig., 2001, pp. 41-44.

[14] P. Cappelletti, C. Golla, P. Olivo, and E. Zanoni, Flash Memories Boston, MA: Kluwer, 1999, pp. 4-6.

[15] A. Kolodny, S. T. K. Nieh, B. Eitan, and J. Shappir, "Analysis and modeling of floating-gate EEPROM cells," IEEE Trans. Electron Devices, vol. ED-33, no. 6, pp. 835-844, Jun. 1986.

[16] C.-K. Baek, B. Kim, Y. Son, W. Kwon, C.-K. Park, Y. J. Park, H. S. Min, and D. M. Kim, "Reliable extraction of cycling induced interface states implementing realistic $\mathrm{P} / \mathrm{E}$ stresses in reference cell: Comparison with Flash memory cell," IEEE Electron Device Lett., vol. 27, no. 3, pp. 169-171, Mar. 2006

[17] D. Esseni, A. D. Strada, P. Cappelletti, and B. Ricco, "A new and flexible scheme for hot-electron programming of nonvolatile memory cells," IEEE Trans. Electron Devices, vol. 46, no. 1, pp. 125-133, Jan. 1999.

[18] Medici User Guide, Synopsys Inc., Mountain View, CA, Jun. 2003.

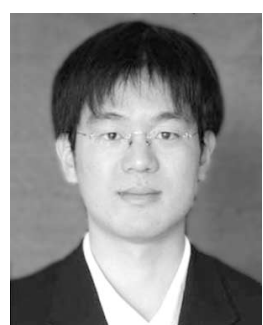

Bomsoo Kim was born in Seoul, Korea, in 1977. He received the B.S. and Ph.D. degrees in electronic and electrical engineering, both from Pohang University of Science and Technology (POSTECH), Pohang, Korea, in 2000 and 2005, respectively.

Currently, he is a Research Fellow of the School of Computational Sciences at Korea Institute for Advanced Study (KIAS). His research interests include the semiconductor device modeling and simulation, especially on the reliability of Flash memory cells.

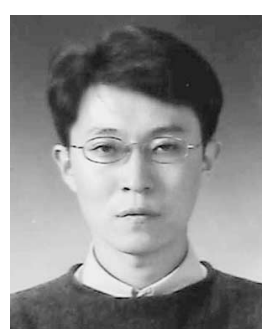

Wook-Hyun Kwon (S'95-M'97) was born in Seoul, Korea, in 1972. He received the B.S. degree in physics from Jung-ang University, Seoul, Korea, in 1995 and the M.S. degree in electrical engineering from Pohang University of Science and Technology (POSTECH), Pohang, Korea, in 1997.

From 1997 to 2002, he was with Hyundai Electronics Industries Company, Ltd., Icheon, Korea, where he developed high-density Flash memories. In 2003, he joined the Samsung Electronics Company, Ltd., Giheung, Korea, where he has been engaged in the research and development of high-density NOR Flash memories. His research interests are device modeling, process integration, design, and reliability for high-density nonvolatile memories.

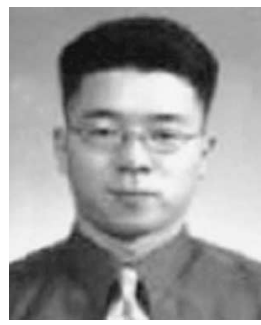

Chang-Ki Baek was born in Seoul, Korea, in 1973 He received the M.S. degree in electronic and electrical engineering from Pohang University of Science and Technology (POSTECH), Pohang, Korea, in 2002. He is currently working toward the Ph.D. degree in electrical engineering and computer science at Seoul National University.

His current research interests include the device analysis and modeling of Flash memory cells.

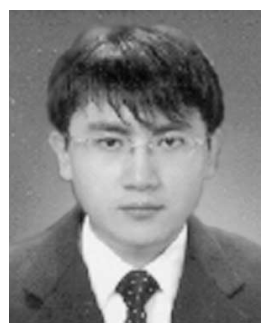

Younghwan Son was born in Daejeon, Korea, in 1981. He received the B.S. degree in electrical engineering from Chungnam National University, Daejeon, in 2004, where he is currently working toward the M.S. degree.

Since 2005, he has been with the School of Computational Sciences, Korea Institute for Advanced Study (KIAS), Seoul, Korea. His research interests include the device analysis and modeling of Flash memory cells, and reliability issues of nano-CMOS devices.

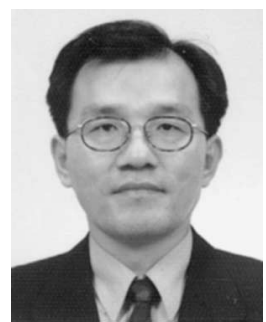

Chan-Kwang Park (S'90-M'92) was born in Incheon, Korea, in 1961. He received the B.S. degree in electronic engineering from Sogang University, Seoul, Korea, in 1984 and the M.S. and Ph.D. degrees in electrical engineering from Korea Advanced Institute of Science and Technology, Daejeon, Korea, in 1987 and 1992, respectively.

From 1992 to 2002, he was with Hyundai Electronics Industries Company, Ltd., Icheon, where he developed high-density dynamic random access memories (DRAMs). In 2003, he joined the Samsung Electronics Company, Ltd., Giheung, Korea, where he engaged in the research and development of high-density NOR Flash memories. His research interests are device modeling, process integration, design, and reliability for high-density nonvolatile memories. 


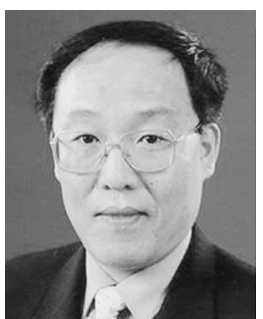

Kinam Kim (S'90-M'97-SM'01-F'03) received the B.Sc. degree in electronic engineering from Seoul National University, Seoul, Korea, in 1981, the M.S. degree in electrical engineering from Korea Advanced Institute of Science and Technology, Seoul, in 1983, and the Ph.D. degree in electrical engineering from the University of California at Los Angeles, in 1994.

In 1983, he joined Samsung Electronics Company, Ltd., Korea, where he was involved in the development of DRAMs, ranging from 64-kb to 4-Gb densities. Currently, he is a Senior Vice President responsible for the research and development of next-generation memory technologies for DRAM, nonvolatile memory, static random access memory, and emerging new memories such as ferroelectric random access memory, phase-change random access memory, and magnetoresistive random access memory. He was a Project Leader for the development of the world's first 1-Gb DRAM using 0.18- $\mu$ m CMOS technologies during 1994-1996. He was a Technical Director for 4-Gb DRAM development during 1998-1999. He was a Program Director for $0.13-\mu \mathrm{m}$ DRAM technology generation from its development at the R\&D center to transference to manufacturing lines during 1999-2001. His current major activity is focused on the development of technologies for low-power and high-performance multigigabit density DRAMs and high-density nonvolatile memories. His research interests are memory device reliability, yield modeling on memory device, memory cell technology, and multilevel metallization for high performance of multigigabit memory devices. He has published more than 260 technical papers in the field of memory technology. He holds 70 patents related to memory technology.

Dr. Kim twice received the Grand Prize of the Samsung group for the successful development of 1-Mb DRAM and 1-Gb DRAM, in 1986 and 1996, respectively. He was a recipient of the Institute for Scientific Information (ISI)'s Citation Award for a highly cited paper. He plays an active part in advancing future memory technology through participating in panel discussions of prime conferences such as the Very Large Scale Integration Technology Symposium. $\mathrm{He}$ served as a committee member of the International Electron Devices Meeting, and he is a member of the editorial advisory board of Microelectronics Reliability. He is a Samsung Fellow.

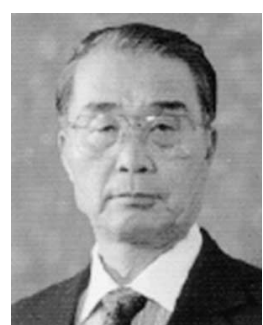

Dae M. Kim received the B.S. degree from Seoul National University, Seoul, Korea, and the M.S. and $\mathrm{Ph} . \mathrm{D}$. degrees from Yale University, New Haven, CT, all in physics.

$\mathrm{He}$ was a Research Associate with the Massachusetts Institute of Technology, Cambridge, and taught at three levels of professorship with the Department of Electrical and Computer Engineering, Rice University, Houston, TX, from 1970 to 1984. After working as a Principal Scientist at Tektronix, Inc., he became a Professor with the Department of Electronics and Electrical Engineering, Pohang University of Science and Technology (POSTECH), serving as the Chairman of the department and the Dean of the Graduate School. Currently, he is a Professor in the Computational Sciences Division, Korea Institute for Advanced Study (KIAS), Seoul. His current research interests are in Flash electrically erasable programmable read-only memory technology, nano-CMOS devices, and Schottky contacts in molecular and CNT devices.

Dr. Kim is a member of American Physical Society and a Fellow of Korean Academy of Science and Technology. 